\title{
Formulae for joint spectral radii of sets of operators
}

\author{
by \\ ViCTOR S. Shulman (Vologda and London) and \\ YURII V. TUROVskII (Baku)
}

\begin{abstract}
The formula $\varrho(M)=\max \left\{\varrho_{\chi}(M), r(M)\right\}$ is proved for precompact sets $M$ of weakly compact operators on a Banach space. Here $\varrho(M)$ is the joint spectral radius (the Rota-Strang radius), $\varrho_{\chi}(M)$ is the Hausdorff spectral radius (connected with the Hausdorff measure of noncompactness) and $r(M)$ is the Berger-Wang radius.
\end{abstract}

1. Notations and preliminaries. In 1960 J.-C. Rota and W. G. Strang [10] defined the joint spectral radius for a bounded set $M$ of operators (or elements of a Banach algebra):

$$
\varrho(M)=\limsup \left\|M^{n}\right\|^{1 / n} .
$$

Here $M^{n}$ denotes the set of all products of $n$ elements of $M$, the norm of a set is the supremum of the norms of its elements. As is well known, since $\|\cdot\|$ is submultiplicative, limsup in (1.1) may be replaced by lim or inf.

This notion has found various applications to operator theory, representation theory of semigroups and Lie algebras, invariant subspaces, geometry of orbits and attractors, evolution dynamics, difference equations, wavelets theory (see [4], [3], [8], [9], [12], [11]). In particular, the importance of the joint spectral radius technique for invariant subspace theory depends primarily on the following simple result: if $\varrho(M)=0$ then all polynomials in elements of $M$ are quasinilpotent (see [11], Corollary 2.10).

For a one-element set $M=\{T\}$, the number $\varrho(M)$ coincides with the usual spectral radius $r(T)=\sup \{|t|: t \in \sigma(T)\}$. For a bounded set $M$ in a Banach algebra, put $r_{\text {sup }}(M)=\sup \{\varrho(T): T \in M\}$. In $1992 \mathrm{M}$. A. Berger and Y. Wang established in [2] that if $M$ is a bounded set of operators on a finite-dimensional space then the norm $\|\cdot\|$ in the definition of $\varrho(M)$ can be replaced by $r_{\text {sup }}(\cdot)$. More transparently, if we define $r(M)=\lim \sup r_{\text {sup }}\left(M^{n}\right)^{1 / n}$, then

$$
\varrho(M)=r(M) \text {. }
$$

2000 Mathematics Subject Classification: Primary 47A13, 47D03, 47A30.

Key words and phrases: joint spectral radius, invariant subspace, Banach algebra. 
We will call (1.2) the Berger-Wang formula and $r(M)$ the Berger-Wang radius of $M$. The formula is important because it relates joint spectral radii to spectra of operators.

It was proved in [11] that (1.2) extends to precompact sets of compact operators on an infinite-dimensional Banach space. To see the convenience of such extension, notice that it easily implies the solution of the Volterra Semigroup Problem: each semigroup of compact quasinilpotent operators has an invariant subspace [12]. Indeed, if a semigroup $G$ consists of compact quasinilpotent operators, then $r(M)=0$ for each finite set $M \subset G$. Hence $\varrho(M)=0$ and all linear combinations of elements of $M$ are quasinilpotent. Thus $G$ is contained in an algebra of quasinilpotent operators and then it has an invariant subspace by the Lomonosov Theorem [7].

The Berger-Wang formula fails in general. P. S. Guinand [6] has constructed a semigroup $G$ of nilpotent operators that contains two operators $T, S$ with nonquasinilpotent $T+S$. Clearly, $\varrho(\{T, S\}) \neq 0, r(\{T, S\})=0$.

It was found in [11] that in some important cases the following "generalized Berger-Wang formula" for precompact $M$ holds:

$$
\varrho(M)=\max \left\{\varrho_{\mathrm{e}}(M), r(M)\right\}=\max \left\{\varrho_{\chi}(M), r(M)\right\}
$$

where $\varrho_{\mathrm{e}}(M)$ is the joint spectral radius of the canonical image of $M$ in the Calkin algebra $B(\mathfrak{X}) / K(\mathfrak{X})$ (called the essential spectral radius) and $\varrho_{\chi}(M)$ is the Hausdorff spectral radius (see the definition below). In particular (1.3) is true if one of the following conditions is valid:

(1) $M$ has no invariant subspaces;

(2) the semigroup $\mathrm{SG}\left(t^{-1} M\right)$ with $t=\varrho(M)>0$ is bounded;

(3) the closed algebra generated by $M$ has no compact operators in its Jacobson radical.

The aim of the present work is to prove (1.3) for any precompact set $M$ of operators on a reflexive Banach space and, more generally, of weakly compact operators on an arbitrary Banach space. In general, for any precompact set $M$ of bounded linear operators on a Banach space, we will establish the other formulae of Berger-Wang type.

In what follows, $\mathfrak{X}_{(1)}$ denotes the unit ball of a Banach space $\mathfrak{X}, B(\mathfrak{X})$ the algebra of all bounded linear operators on $\mathfrak{X}, K(\mathfrak{X})$ the ideal of compact operators, $\pi_{K}$ the canonical surjection from $B(\mathfrak{X})$ onto $B(\mathfrak{X}) / K(\mathfrak{X})$, $\|T \mid\|=\left\|\pi_{K}(T)\right\|$ the essential norm of an operator $T \in B(\mathfrak{X})$. Clearly, $\|T\| \|$ can be regarded as a measure of noncompactness of $T$; we will also need another measure of noncompactness $\|T\|_{\chi}=\chi\left(T \mathfrak{X}_{(1)}\right)$, where $\chi(E)$ for a bounded set $E$ means the infimum of all $\varepsilon$ such that $E$ contains a finite $\varepsilon$-net. Clearly, $\|T\|_{\chi} \leq\|T\|$ and $\|T\|_{\chi}=0$ if and only if $T \in K(\mathfrak{X})$. The advantage of the submultiplicative seminorm $\|\cdot\|_{\chi}$ is that it cannot grow 
if we pass to restrictions or quotients of operators; this is not quite clear for $\||\cdot|\|$.

The restriction of an operator $T$ to an invariant subspace $Y$ is denoted by $T \mid Y$. Similarly, if $Y_{1} \subset Y_{2}$ are $T$-invariant subspaces then $T \mid\left(Y_{2} / Y_{1}\right)$ is the operator induced by $T$ on the quotient space $Y_{2} / Y_{1}$.

Let $M$ denote a set of operators. The individual characteristics $\|T\|$, $\|T\|\|\|, T \|_{\chi}$ extend to $M$ via supremum: $\|M\|_{\chi}=\sup \left\{\|T\|_{\chi}: T \in M\right\}$ and so on. We say that $M$ is Hausdorff-bounded if $\|M\|_{\chi}<\infty$. Similarly to $r$ (with respect to $r_{\text {sup }}$ ), $\varrho$ (with respect to the usual norm), $\varrho_{\mathrm{e}}$ (with respect to the essential norm) we define the Hausdorff spectral radius $\varrho_{\chi}$ for a Hausdorff-bounded set $M$ as follows:

$$
\varrho_{\chi}(M)=\lim \sup \left\|M^{n}\right\|_{\chi}^{1 / n} .
$$

A chain is any set of closed subspaces of $\mathfrak{X}$ linearly ordered by inclusion. A nest is a chain which is complete with respect to inf and sup and contains $(0)$ and $\mathfrak{X}$. A gap in a chain $\Gamma$ is a pair $Y \subset Z$ of subspaces without intermediate subspaces in $\Gamma$. The space $Z / Y$ is called a gap-quotient and is usually denoted by $Z^{\sim}$ (it is completely determined by $Z$ ). The set of all gap-quotients for $\Gamma$ is denoted by $\operatorname{gap}(\Gamma)$; if $\operatorname{gap}(\Gamma)$ is empty, $\Gamma$ is said to be continuous. Each continuous nest is maximal (it is not contained in a greater nest); more generally, a nest is maximal iff its gap-quotients are one-dimensional.

The lattice of all $M$-invariant subspaces is denoted by lat $M$. If $Y \in$ lat $M$ we write $M \mid Y=\{T \mid Y: T \in M\}$ and similarly for quotients. If $\Gamma$ is a set of closed subspaces of $\mathfrak{X}$, then alg $\Gamma$ denotes the algebra of all operators $T \in B(\mathfrak{X})$ such that $\Gamma \subset$ lat $T$. Given an operator $T \in B(\mathfrak{X})$ and a subspace $Z \subset \mathfrak{X}$, we write $T Z_{(1)}$ for $T\left(Z_{(1)}\right) \equiv\left\{T x: x \in Z_{(1)}\right\}$.

If $\Gamma \subset$ lat $M$ is a chain, we set

$$
\begin{aligned}
\|M \mid \Gamma\| & =\sup \{\|T \mid V\|: V \in \operatorname{gap}(\Gamma)\}, \\
\varrho & (M \mid \Gamma)=\sup \{\varrho(M \mid V): V \in \operatorname{gap}(\Gamma)\} ;
\end{aligned}
$$

both values are assumed to be zero if $\Gamma$ is continuous. We also define $\varrho(M \mid \Gamma)$ as follows: $\varrho(M \mid \Gamma)=\lim \sup \left\|M^{n} \mid \Gamma\right\|^{1 / n}$. Since $\|\cdot \mid \Gamma\|$ is a submultiplicative seminorm on $\operatorname{alg} \Gamma$, as above we obtain $\varrho(M \mid \Gamma)=\lim \left\|M^{n} \mid \Gamma\right\|^{1 / n}=$ $\inf \left\|M^{n} \mid \Gamma\right\|^{1 / n}$. It is clear that $\widehat{\varrho}(M \mid \Gamma) \leq \varrho(M \mid \Gamma)$.

We need the following results of [11].

Lemma 1.1 ([11], Corollary 4.3). If $M \subset B(\mathfrak{X})$ is bounded and $F \subset$ lat $M$ is a finite nest, then $\varrho(M)=\widehat{\varrho}(M \mid F)$.

Given a Banach space $\mathfrak{X}$, a set $G \subset \mathfrak{X}$ and a closed subspace $Y \subset \mathfrak{X}$, $G / Y$ denotes the image of $G$ under the canonical map $\mathfrak{X} \rightarrow \mathfrak{X} / Y$ (we adopt this notation to avoid the confusion with $G+Y$; here we understand $G+Y$ as a sum of two sets, namely $G+Y=\{x+y: x \in G, y \in Y\})$. 
Lemma 1.2 ([11], Lemma 6.9). Let $G$ be a precompact subset of a $B a-$ nach space $\mathfrak{X}$, and let $Y$ be a closed subspace of $\mathfrak{X}$. Then for any $\varepsilon>0$ there exist precompact sets $G_{1} \subset \mathfrak{X}$ and $G_{2} \subset Y$ such that $G \subset G_{1}+G_{2}$ and $\left\|G_{1}\right\| \leq\|G / Y\|+\varepsilon$.

Recall that $M \subset B(\mathfrak{X})$ is irreducible if lat $M$ is trivial.

Lemma 1.3 ([11], Theorem 9.4). If $M \subset B(\mathfrak{X})$ is irreducible and precompact, then $\varrho(M)=\max \left\{\varrho_{\chi}(M), r(M)\right\}$.

2. Auxiliary lemmas. As a rule, in what follows, $M$ denotes a set of operators on a Banach space $\mathfrak{X}$.

Lemma 2.1. If $M$ is precompact then $\|M\|_{\chi}=\chi\left(M \mathfrak{X}_{(1)}\right)$.

Proof. Since $T \mathfrak{X}_{(1)} \subset M \mathfrak{X}_{(1)}$ for $T \in M$, the inequality $\leq$ is evident. Let $\|M\|_{\chi} \leq \alpha$. For $\varepsilon>0$ choose an $\varepsilon$-net $T_{1}, \ldots, T_{n}$ in $M$ and for any $j \leq n$ a finite $\alpha$-net in $T_{j} \mathfrak{X}_{(1)}$; their union will be a finite $(\alpha+\varepsilon)$-net in $M \mathfrak{X}_{(1)}$. Thus $\chi\left(M \mathfrak{X}_{(1)}\right) \leq \alpha+\varepsilon$; taking the infimum we obtain the inequality $\geq$.

Lemma 2.2. Let $M$ be precompact, and let $\Gamma \subset$ lat $M$ be an infinite chain of nonzero subspaces with zero intersection. Then for any $\alpha>\|M\|_{\chi}$ there exists $Z_{0}$ in $\Gamma$ such that $\left\|M \mid\left(Z_{0} / Z\right)\right\| \leq 2 \alpha$ for all $Z \subset Z_{0}$ in $\Gamma$.

Proof. Note first that the interval $((0), Z]_{\Gamma} \equiv\{Y \in \Gamma: Y \subset Z\}$ contains an infinite number of elements for any $Z \subset \Gamma$. So, if the assertion is not valid then there exists a decreasing sequence $\left(Z_{n}\right)$ in $\Gamma$ such that

$$
\left\|M \mid\left(Z_{n} / Z_{n+1}\right)\right\|>2 \alpha .
$$

Hence there are $x_{n}$ in $\left(Z_{n}\right)_{(1)}$ and $T_{n}$ in $M$ with $\left\|T_{n} x_{n}-y\right\|>2 \alpha$ for any $y$ in $Z_{n+1}$. It follows that

$$
\left\|T_{n} x_{n}-T_{k} x_{k}\right\|>2 \alpha
$$

for $n \neq k$. This implies that $M \mathfrak{X}_{(1)}$ does not contain a finite $\alpha$-net, in contradiction to Lemma 2.1.

The proof of Lemma 2.2 actually establishes the following result.

Lemma 2.3. If $M$ is precompact and $\alpha>\|M\|_{\chi}$, then any chain $\Gamma \subset$ lat $M$ has only a finite number of gap-quotients $V \in \operatorname{gap}(\Gamma)$ with $\|M \mid V\|$ $\geq 2 \alpha$.

Proof. Indeed, if not, then there exists an infinite set of gaps $\left(Z_{n}, Y_{n}\right)$ with

$$
\left\|M \mid\left(Y_{n} / Z_{n}\right)\right\| \geq 2 \alpha .
$$

Hence there exists an infinite sequence $\left(T_{n} x_{n}\right)$ with $x_{n} \in\left(Y_{n}\right)_{(1)}, T_{n} \in M$ and with property (2.1), a contradiction. 
Lemma 2.4. If $M$ is precompact and $\varrho_{\chi}(M)<\varrho(M \mid \Gamma)$ for a chain $\Gamma \subset$ lat $M$ then $\varrho(M \mid \Gamma)=\widehat{\varrho}(M \mid \Gamma)$. In other words, $\varrho(M \mid \Gamma) \leq \max \left\{\varrho_{\chi}(M)\right.$, $\widehat{\varrho}(M \mid \Gamma)\}$.

Proof. Suppose that $\varrho(M \mid \Gamma)=1$ and $\varrho_{\chi}(M)<\alpha<\beta<1$ for some $\alpha, \beta$. Then there is a number $n$ such that $\left\|M^{n}\right\|_{\chi} \leq \alpha^{n} \leq \beta^{n} / 2$. By Lemma 2.3 , the set

$$
G_{1}=\left\{V \in \operatorname{gap}(\Gamma):\left\|M^{n} \mid V\right\| \geq 2 \alpha^{n}\right\}
$$

is finite. Given $G \subset \operatorname{gap}(\Gamma)$, let

$$
\|M \mid G\|=\sup \left\{\left\|M^{n} \mid V\right\|: V \in G\right\} .
$$

Note that $\|\cdot \mid G\|$ is a submultiplicative seminorm on $\operatorname{alg} \Gamma$, so that the limit $\lim \left\|M^{m} \mid G\right\|^{1 / m}$ exists and $\left\|M^{n m}\left|G\left\|^{1 /(n m)} \leq\right\| M^{n}\right| G\right\|^{1 / n}$ for each $m>0$.

Put $G_{2}=\operatorname{gap}(\Gamma) \backslash G_{1}$. Then, for every $m>0$,

$$
\left\|M^{n m}\left|G_{2}\left\|^{1 /(n m)} \leq\right\| M^{n}\right| G_{2}\right\|^{1 / n} \leq \beta .
$$

Since

$$
\begin{aligned}
\varrho(M \mid \Gamma) & =\lim _{m}\left\|M^{n m}\left|\Gamma\left\|^{1 /(n m)}=\lim _{m}\right\| M^{n m}\right|\left(G_{1} \cup G_{2}\right)\right\|^{1 /(n m)} \\
& =\max \left\{\lim _{m}\left\|M^{n m}\left|G_{1}\left\|^{1 /(n m)}, \lim _{m}\right\| M^{n m}\right| G_{2}\right\|^{1 /(n m)}\right\},
\end{aligned}
$$

we obtain

$$
\varrho(M \mid \Gamma)=\max \left\{\lim _{m}\left\|M^{n m} \mid G_{1}\right\|^{1 /(n m)}, \beta\right\} .
$$

Since $\beta<1, \varrho(M \mid \Gamma)=\lim _{m}\left\|M^{n m} \mid G_{1}\right\|^{1 /(n m)}$. Since $G_{1}$ is finite,

$$
\varrho(M \mid \Gamma)=\lim _{m}\left\|M^{n m} \mid G_{1}\right\|^{1 /(n m)}=\max \left\{\varrho(M \mid V): V \in G_{1}\right\} \leq \widehat{\varrho}(M \mid \Gamma),
$$

whence $\varrho(M \mid \Gamma)=\widehat{\varrho}(M \mid \Gamma)$.

Lemma 2.5. Let $M \subset B(\mathfrak{X})$ be precompact, and let $Y, Z \in$ lat $M$ with $Z \subset Y$. Then $\left\|M\left|Y\left\|_{\chi} \leq 2\right\| M\left\|_{\chi},\right\| M\right|(Y / Z)\right\|_{\chi} \leq 2\|M\|_{\chi}$ and $\varrho_{\chi}(M \mid(Y / Z))$ $\leq \varrho_{\chi}(M)$. Moreover, $\|M \mid(\mathfrak{X} / Z)\|_{\chi} \leq\|M\|_{\chi}$.

Proof. If $M Y_{(1)}$ has a finite $\alpha$-net in $\mathfrak{X}$ then it clearly has a finite $2 \alpha$-net in $Y$. So, by Lemma 2.1,

$$
\|M \mid Y\|_{\chi}=\chi\left((M \mid Y) Y_{(1)}\right) \leq 2 \chi\left(M Y_{(1)}\right) \leq 2 \chi\left(M \mathfrak{X}_{(1)}\right)=2\|M\|_{\chi} .
$$

Since images of $\varepsilon$-nets under the canonical map $Y \rightarrow Y / Z$ are $\varepsilon$-nets, we easily obtain

$$
\left\|M\left|(Y / Z)\left\|_{\chi}=\chi\left(M(Y / Z)_{(1)}\right) \leq \chi\left((M \mid Y) Y_{(1)}\right)=\right\| M\right| Y\right\|_{\chi} .
$$

Hence $\|M \mid(Y / Z)\|_{\chi} \leq 2\|M\|_{\chi}$ and also $\|M \mid(\mathfrak{X} / Z)\|_{\chi} \leq\|M\|_{\chi}$.

Now $\varrho_{\chi}(M \mid(Y / Z))=\lim \left\|M^{n} \mid(Y / Z)\right\|_{\chi}^{1 / n} \leq \lim 2^{1 / n}\left\|M^{n}\right\|_{\chi}^{1 / n}=\varrho_{\chi}(M)$. 
Let $F$ be a complete lattice of closed subspaces of $\mathfrak{X}$, and let $\Gamma \subset F$ be a chain. We say that $\Gamma$ is relatively maximal in $F$ if $\Gamma \subset \Gamma_{0}$ for a chain $\Gamma_{0} \subset F$ implies $\Gamma_{0}=\Gamma$.

Proposition 2.6. Let $M$ be precompact. If there exists a finite, relatively maximal nest $\Gamma \subset$ lat $M$, then $\varrho(M)=\max \left\{\varrho_{\chi}(M), r(M)\right\}$.

Proof. This follows from Lemmas 1.1, 1.3, 2.5 and the obvious inequality $r(M \mid(Y / Z)) \leq r(M)$ for $Y, Z \in$ lat $M$ with $Z \subset Y$.

So, to obtain the general analogs of Proposition 2.6, we may consider only the case of infinite nests.

Lemma 2.7. If $Q, N$ are bounded subsets of a Banach algebra $A$ with

$$
[Q, N] \equiv\{a b-b a: a \in Q, b \in N\}=\{0\},
$$

then $|\varrho(Q)-\varrho(N)| \leq \operatorname{dist}(Q, N)$ (dist here is the Hausdorff distance).

Proof. Let $\operatorname{dist}(Q, N)<\varepsilon$ and $\varrho(N)<\alpha$; it suffices to prove that $\varrho(Q)<$ $\alpha+\varepsilon$. It follows easily from the definition of $\varrho$ that there exists a constant $C$ with $\left\|N^{k}\right\|<C \alpha^{k}$ for all $k$. Let $a_{1}, \ldots, a_{n}$ belong to $Q$, and let us find $b_{1}, \ldots, b_{n}$ in $N$ with $\left\|a_{i}-b_{i}\right\|<\varepsilon$. Setting $c_{i}=a_{i}-b_{i}$, we have $a_{1} \ldots a_{n}=$ $\left(b_{1}+c_{1}\right) \ldots\left(b_{n}+c_{n}\right)=d_{0}+\ldots+d_{n}$, where $d_{k}$ is a sum of $\left(\begin{array}{l}n \\ k\end{array}\right)$ elements that are products of $k$ elements of $\left\{c_{i}\right\}$ and $n-k$ elements of $\left\{b_{i}\right\}$. Hence $\left\|d_{k}\right\| \leq\left(\begin{array}{l}n \\ k\end{array}\right) \varepsilon^{k}\left\|N^{n-k}\right\| \leq\left(\begin{array}{l}n \\ k\end{array}\right) \varepsilon^{k} C \alpha^{n-k}$ and $\left\|a_{1} \ldots a_{n}\right\| \leq C(\alpha+\varepsilon)^{n}$. Thus $\left\|Q^{n}\right\|^{1 / n} \leq C^{1 / n}(\alpha+\varepsilon)$ and $\varrho(Q) \leq \alpha+\varepsilon$.

Lemma 2.8. For a precompact set $N$ of commuting elements of a Banach algebra, $\varrho(N)=r(N)=r_{\text {sup }}(N)$.

Proof. If $N$ is finite the result follows by a direct computation. In the general case take a finite $\varepsilon$-net $Q$ in $N$; then $\operatorname{dist}(Q, N)<\varepsilon$ and by Lemma 2.7, $\varrho(N) \leq \varrho(Q)+\varepsilon \leq r_{\text {sup }}(Q)+\varepsilon \leq r_{\text {sup }}(N)+\varepsilon$. Since $\varepsilon$ is arbitrary, $\varrho(N) \leq r_{\text {sup }}(N)$. The inequality $r_{\text {sup }}(N) \leq \varrho(N)$ is evident.

3. Using the weakly compact operators. Let $W(\mathfrak{X})$ denote the set of all weakly compact operators on a Banach space $\mathfrak{X}$ (see, for example, [5], Section 3.3). As is known, $W(\mathfrak{X})$ is a closed ideal of $B(\mathfrak{X})$ and $K(\mathfrak{X}) \subset W(\mathfrak{X})$.

Let $\Gamma$ be a chain of closed subspaces in $\mathfrak{X}$, and let $W(\Gamma)=\operatorname{alg} \Gamma \cap W(\mathfrak{X})$. Then $W(\Gamma)$ is a closed ideal of $\operatorname{alg} \Gamma$ and $\operatorname{alg} \Gamma / W(\Gamma)$ is a Banach algebra. Given an operator $T \in \operatorname{alg} \Gamma$ or a set $N \subset \operatorname{alg} \Gamma$, we write for brevity $\|T\|_{w, \Gamma}$ instead of $\|T / W(\Gamma)\|=\inf \{\|T+S\|: S \in W(\Gamma)\}$ and $\|N\|_{w, \Gamma}$ instead of $\|N / W(\Gamma)\|=\sup \left\{\|T\|_{w, \Gamma}: T \in N\right\}$.

Lemma 3.1. Let $M$ be a precompact set, $\Gamma \subset$ lat $M$ a chain, and $\Gamma_{0} \subset \Gamma$ a subchain of nonzero subspaces with zero intersection. Then, for any $\varepsilon>0$, 
one can find $Z$ in $\Gamma_{0}$ with

$$
\left\|M^{2} \mid Z\right\| \leq 4\|M\| \max \left\{\|M\|_{w, \Gamma},\|M\|_{\chi}\right\}+\varepsilon
$$

Proof. One may suppose that $\|M\|>0$. It suffices to obtain the result for a finite $\varepsilon_{0}$-net $M_{\varepsilon_{0}} \subset M$ and, moreover, for any $T_{1}, T_{2}$ in $M_{\varepsilon_{0}}$, to find $Z$ in $\Gamma_{0}$ satisfying the condition

$$
\left\|T_{1} T_{2} \mid Z\right\| \leq 4\|M\| \max \left\{\|M\|_{\chi},\|M\|_{w, \Gamma}\right\}+\varepsilon / 2
$$

and then to take the intersection, say $Y$, of such subspaces for all pairs. Indeed, if $\varepsilon_{0}<\varepsilon(4\|M\|)^{-1}$ then the obvious inequality $\left\|M^{2}\left|Y\|\leq\| M_{\varepsilon_{0}}^{2}\right| Y\right\|+$ $\varepsilon / 2$ and (3.1) with $Z=Y$ complete the proof. In other words, the proof is reduced to the case of a finite set and it suffices to show (3.1) for any $T_{1}, T_{2} \in M$.

By Lemma 2.2, for $\varepsilon_{1}>0$, there exists $Z_{0}$ in $\Gamma_{0}$ such that

$$
\left\|T_{2} \mid\left(Z_{0} / Z\right)\right\| \leq 2\left(\|M\|_{\chi}+\varepsilon_{1}\right)
$$

for all $Z \subset Z_{0}$ in $\Gamma_{0}$. Hence, for any $x$ in $\left(Z_{0}\right)_{(1)}$ and for any $Z \subset Z_{0}$ in $\Gamma_{0}$, one can choose $y=y(x, Z)$ in $Z$ with $\left\|T_{2} x-y(x, Z)\right\| \leq 2\left(\|M\|_{\chi}+\varepsilon_{1}\right)$. Set $\alpha_{1}=2\left(\|M\|_{\chi}+\varepsilon_{1}\right)$. Then

$$
\|y(x, Z)\| \leq\left\|T_{2} x-y(x, Z)\right\|+\left\|T_{2} x\right\| \leq \alpha_{1}+\|M\|
$$

and

$$
\left\|T_{1} T_{2} x-T_{1} y(x, Z)\right\| \leq 2\left\|T_{1}\right\|\left(\|M\|_{\chi}+\varepsilon\right) \leq \alpha_{1}\|M\| .
$$

It follows from Lemma 1.2 that, for $\varepsilon_{2}>0$, there exist precompact sets $M_{1} \subset \operatorname{alg} \Gamma$ and $M_{2} \subset W(\Gamma)$ such that $\left\|M_{1}\right\| \leq\|M\|_{w, \Gamma}+\varepsilon_{2}$ and $M \subset M_{1}+M_{2}$. Therefore $T_{1}=S_{1}+S_{2}$ for some $S_{1} \in M_{1}$ and $S_{2} \in M_{2}$. Set $\alpha_{2}=\|M\|_{w, \Gamma}+\varepsilon_{2}$. It follows from (3.2), (3.3) and the inequality $\left\|S_{1}\right\| \leq \alpha_{2}$ that

$$
\begin{aligned}
\left\|T_{1} T_{2} x-S_{2} y(x, Z)\right\| & \leq\left\|T_{1} T_{2} x-T_{1} y(x, Z)\right\|+\left\|S_{1} y(x, Z)\right\| \\
& \leq \alpha_{1}\|M\|+\left\|S_{1}\right\| \cdot\|y(x, Z)\| \\
& \leq \alpha_{1}\|M\|+\alpha_{2}\left(\alpha_{1}+\|M\|\right) \\
& \leq\left(\alpha_{1}+\alpha_{2}\right)\|M\|+\alpha_{1} \alpha_{2} .
\end{aligned}
$$

Since $\alpha_{1} \leq 2\|M\|\left(1+\varepsilon_{1}\|M\|^{-1}\right)$ and $\alpha_{2} \leq\|M\|\left(1+\varepsilon_{2}\|M\|^{-1}\right)$, we obtain $\alpha_{1} \alpha_{2} \leq \sqrt{2 \alpha_{1} \alpha_{2}}\|M\|\left(1+\varepsilon_{3}\right)$, where

$$
1+\varepsilon_{3}=\sqrt{\left(1+\varepsilon_{1}\|M\|^{-1}\right)\left(1+\varepsilon_{2}\|M\|^{-1}\right)} \leq \sqrt{2}
$$

if $\varepsilon_{1}$ and $\varepsilon_{2}$ are small enough. Therefore $\alpha_{1} \alpha_{2} \leq 2 \sqrt{\alpha_{1} \alpha_{2}}\|M\|$ and

$$
\begin{aligned}
\left(\alpha_{1}+\alpha_{2}\right)\|M\|+\alpha_{1} \alpha_{2} & \leq\left(\sqrt{\alpha_{1}}+\sqrt{\alpha_{2}}\right)^{2}\|M\| \\
& \leq\left(2 \max \left\{\sqrt{\alpha_{1}}, \sqrt{\alpha_{2}}\right\}\right)^{2}\|M\| \\
& \leq 4\|M\| \max \left\{\alpha_{1}, \alpha_{2}\right\} .
\end{aligned}
$$


It follows from (3.4) and (3.5) that

$$
\left\|T_{1} T_{2} x-S_{2} y(x, Z)\right\| \leq 4\|M\| \max \left\{\alpha_{1}, \alpha_{2}\right\} .
$$

Since the set $\left\{y(x, Z): Z \subset Z_{0}, Z \in \Gamma_{0}\right\}$ is bounded (see (3.2)), $S_{2}$ is weakly compact and $D=\left\{Z \in \Gamma_{0}: Z \subset Z_{0}\right\}$ is a directed set (with respect to $\subset$ ), we deduce that the net $\left(S_{2} y(x, Z)\right)_{Z \in D}$ has a weak limit point, say $z$. Since $S_{2} y(x, Z) \in Z$ for each subspace $Z \in D$ which is weakly closed, the point $z$ must belong to all $Z$ in $\Gamma_{0}$, hence must be zero. So $T_{1} T_{2} x$ is a weak limit point of the net $Z \mapsto T_{1} T_{2} x-S_{2} y(x, Z)$. Then

$$
\left\|T_{1} T_{2} x\right\| \leq \liminf \left(\left\|T_{1} T_{2} x-S_{2} y(x, Z)\right\|\right)_{Z \in D},
$$

and it follows from (3.6) that $\left\|T_{1} T_{2} x\right\| \leq 4\|M\| \max \left\{\alpha_{1}, \alpha_{2}\right\}$. Since $x$ in $\left(Z_{0}\right)_{(1)}$ is arbitrary,

$$
\left\|T_{1} T_{2} \mid Z_{0}\right\| \leq 4\|M\| \max \left\{\alpha_{1}, \alpha_{2}\right\} .
$$

Now $Z_{0}$ is a subspace we looked for (i.e., $Z=Z_{0}$ satisfies (3.1)) if $\max \left\{\varepsilon_{1}, \varepsilon_{2}\right\}$ $\leq \varepsilon(8\|M\|)^{-1}$.

LEMMA 3.2. Let $\Gamma$ be a chain of closed subspaces in $\mathfrak{X}$ and $Z, Y \in \Gamma$ with $Z \subset Y$. Let $\Gamma \mid(Y / Z) \equiv\{V / Z: V \in \Gamma, Z \subset V \subset Y\}$. If $N \subset$ alg $\Gamma$ then $N \mid(Y / Z) \subset \operatorname{alg}(\Gamma \mid(Y / Z))$ and $\|N \mid(Y / Z)\|_{w, \Gamma \mid(Y / Z)} \leq\|N\|_{w, \Gamma}$.

Proof. Set $F=\Gamma \mid(Y / Z)$. For $\varepsilon>0$, let $T \in N$ be arbitrary, and choose $S \in W(\Gamma)$ with $\|T\|_{w, \Gamma} \geq\|T+S\|-\varepsilon$. Note that $\Gamma \subset \operatorname{lat}\{T, S\}$, and standard arguments show that $S \mid(Y / Z)$ is weakly compact. Hence $T \mid(Y / Z) \in$ $\operatorname{alg} F$ and $S \mid(Y / Z) \in W(F)$. So

$$
\left\|T\left|(Y / Z)\left\|_{w, F} \leq\right\|(T+S)\right|(Y / Z)\right\| \leq\|T+S\| \leq\|T\|_{w, \Gamma}+\varepsilon .
$$

Taking suprema, we obtain $\|N \mid(Y / Z)\|_{w, F} \leq\|N\|_{w, \Gamma}+\varepsilon$ and therefore

$$
\|N \mid(Y / Z)\|_{w, F} \leq\|N\|_{w, \Gamma} \text {. }
$$

Lemma 3.3. Let $M$ be a precompact set and $\Gamma$ a nest in lat $M$. Then for any $\varepsilon>0$ there exists a finite subnest $F \subset \Gamma$ such that

$$
\left\|M^{2} \mid F\right\| \leq \max \left\{4\|M\| \cdot\|M\|_{w, \Gamma}, 4\|M\| \cdot\|M\|_{\chi},\left\|M^{2} \mid \Gamma\right\|\right\}+\varepsilon
$$

Proof. Denote by $4 \alpha$ the right hand side of (3.7). As above (see the beginning of the proof of Lemma 3.1), the problem reduces to the case of finite $M$ and, moreover, it suffices to find, for any $T \in M^{2}$, a finite subnest $F$ of $\Gamma$ with $\|T \mid F\| \leq 4 \alpha$. The union of such finite subnests (when $T$ runs over the finite set $M$ ) is required for the completeness of the proof.

Since $\alpha>\|M\| \cdot\|M\|_{\chi} \geq\left\|M^{2}\right\|_{\chi} \geq\|T\|_{\chi}$, there exists by Lemma 2.1 a finite $\alpha$-net $E=\left\{u_{1}, \ldots, u_{n}\right\}$ in the closure of $T \mathfrak{X}_{(1)}$. For $Z \in \Gamma$, let

$$
g(Z)=\left\{u_{j} \in E: \operatorname{dist}\left(u_{j}, T Z_{(1)}\right) \leq \alpha\right\}
$$


(here, as usual, $\left.\operatorname{dist}\left(u_{j}, T Z_{(1)}\right)=\inf \left\{\left\|u_{j}-x\right\|: x \in T Z_{(1)}\right\}\right)$. Then

$$
\operatorname{dist}\left(T Z_{(1)}, g(Z)\right) \leq \alpha
$$

where we also use dist to denote the Hausdorff distance on the set of all bounded subsets of $\mathfrak{X}$. Indeed, $\operatorname{dist}\left(u, T Z_{(1)}\right) \leq \alpha$ for each $u$ in $g(Z)$ by the definition. Furthermore, for any $y \in T Z_{(1)}$ there exists $u_{j} \in E$ with $\left\|y-u_{j}\right\| \leq \alpha$. Hence $u_{j} \in g(Z)$ and $\operatorname{dist}(y, g(Z)) \leq \alpha$.

The set of all subsets $g(Z)$ of $E$ is finite and linearly ordered by inclusion. So it can be enumerated increasingly: $E_{1} \subset \ldots \subset E_{m}$. Clearly, $E_{1}=g((0))$, $E_{m}=g(\mathfrak{X})$. Set $\Gamma_{j}=\left\{Z \in \Gamma: g(Z)=E_{j}\right\}$ for any $j, 1 \leq j \leq m$. Then $\Gamma$ is the disjoint union of $\Gamma_{j}$.

Let $Y, Z$ belong to the same $\Gamma_{j}$. It follows easily from (3.8) that

$$
\operatorname{dist}\left(T Y_{(1)}, T Z_{(1)}\right) \leq 2 \alpha \text {. }
$$

One may suppose that $Z \subset Y$. Hence

$$
\begin{aligned}
\|T \mid(Y / Z)\| & =\sup \left\{\|T z / Y\|: z \in Z_{(1)}\right\} \\
& \leq \sup \left\{\operatorname{dist}\left(T z, T Y_{(1)}\right): z \in Z_{(1)}\right\} \\
& \leq \operatorname{dist}\left(T Z_{(1)}, T Y_{(1)}\right) \leq 2 \alpha .
\end{aligned}
$$

Let us examine the "boundary" subspaces. Let $\mathfrak{X}_{j}^{+}=\bigcap\left\{Z: Z \in \Gamma_{j+1}\right\}$, and let $\mathfrak{X}_{j}^{-}$be the closure of $\bigcup\left\{Z: Z \in \Gamma_{j}\right\}$. We obtain a finite sequence of subspaces $\mathfrak{X}_{0}^{+}=(0), \mathfrak{X}_{1}^{-}, \mathfrak{X}_{1}^{+}, \ldots, \mathfrak{X}_{m-1}^{-}, \mathfrak{X}_{m-1}^{+}, \mathfrak{X}_{m}^{-}=\mathfrak{X}$.

Set, for convenience, $\mathfrak{X}_{0}^{-}=(0), \Gamma_{0}=\left\{\mathfrak{X}_{0}^{-}\right\}$and $\mathfrak{X}_{m}^{+}=\mathfrak{X}$.

It is clear that $\mathfrak{X}_{j}^{-} \subset \mathfrak{X}_{j}^{+}$. If they do not coincide then $\mathfrak{X}_{j}^{-} \in \Gamma_{j}, \mathfrak{X}_{j}^{+} \in$ $\Gamma_{j+1}$ and $\mathfrak{X}_{j}^{+} / \mathfrak{X}_{j}^{-} \in \operatorname{gap}(\Gamma)$, whence

$$
\left\|T\left|\left(\mathfrak{X}_{j}^{+} / \mathfrak{X}_{j}^{-}\right)\|\leq\| T\right| \Gamma\right\| \leq\left\|M^{2} \mid \Gamma\right\|<4 \alpha .
$$

Assume now that $\mathfrak{X}_{j}^{-}=\mathfrak{X}_{j}^{+}$for some $j, 0 \leq j \leq m$, and denote them by $\mathfrak{X}_{j}$. If $j=m$ then it follows from (3.9) that $\left\|T \mid\left(\mathfrak{X}_{m} / Z\right)\right\| \leq 2 \alpha$ for any $Z \in \Gamma_{m}$ (because $\mathfrak{X}_{m}=\mathfrak{X} \in \Gamma_{m}$ ). So one may suppose that $j<m$. If $\mathfrak{X}_{j}$ in $\Gamma_{j+1}$ then $g\left(\mathfrak{X}_{j}\right)=E_{j+1}$. It follows that for any $y \in T\left(\mathfrak{X}_{j}\right)_{(1)}$ we have $y=\lim y_{n}$ with $y_{n} \in T\left(Z_{n}\right)_{(1)}, Z_{n} \in \Gamma_{j}$ and $\operatorname{dist}\left(y_{n}, E_{j}\right) \leq \alpha$. Hence $\operatorname{dist}\left(y, E_{j}\right) \leq \alpha, \operatorname{dist}\left(T\left(\mathfrak{X}_{j}\right)_{(1)}, T Z_{(1)}\right) \leq 2 \alpha$ and, as above,

$$
\left\|T \mid\left(\mathfrak{X}_{j} / Z\right)\right\| \leq 2 \alpha
$$

for any $Z$ in $\Gamma_{j}$. Note that, in this case, if $j=m-1$, we also have

$$
\left\|T \mid\left(\mathfrak{X}_{m} / \mathfrak{X}_{m-1}\right)\right\| \leq 2 \alpha .
$$

Let $\mathfrak{X}_{j}$ belong to $\Gamma_{j}$ for $j<m$, and let $V=\mathfrak{X} / \mathfrak{X}_{j}$. Then the chain

$$
\Gamma_{j+1} \mid V=\left\{Z / \mathfrak{X}_{j}: Z \in \Gamma_{j+1}, Z \supset \mathfrak{X}_{j}\right\}
$$

is a chain of nonzero subspaces with zero intersection. Applying Lemma 3.1 to the space $V$, the operator $T \mid V$ and the chain $\Gamma_{j+1}|V \subset \Gamma| V$, we obtain 
$Z$ in $\Gamma_{j+1}$ with

$$
\left\|T\left|\left(Z / \mathfrak{X}_{j}\right)\|\leq 4\| M\right| V\right\| \max \left\{\left\|M\left|V\left\|_{w, \Gamma \mid V},\right\| M\right| V\right\|_{\chi}\right\}+\varepsilon .
$$

We denote the subspace $Z$ by $Z_{j}$.

It is clear that $\|M \mid V\| \leq\|M\|$. By Lemmas 3.2 and 2.5, $\|M \mid V\|_{w, \Gamma \mid V} \leq$ $\|M\|_{w, \Gamma}$ and $\|M \mid V\|_{\chi} \leq\|M\|_{\chi}$. Then it follows from (3.11) that

$$
\left\|T \mid\left(Z_{j} / \mathfrak{X}_{j}\right)\right\| \leq 4\|M\| \max \left\{\|M\|_{w, \Gamma},\|M\|_{\chi}\right\}+\varepsilon \leq 4 \alpha .
$$

Denote by $F$ the chain consisting of all boundary subspaces $\mathfrak{X}_{0}^{+}, \mathfrak{X}_{1}^{-}, \mathfrak{X}_{1}^{+}$, $\ldots, \mathfrak{X}_{m-1}^{-}, \mathfrak{X}_{m-1}^{+}, \mathfrak{X}_{m}^{-}$and all subspaces $Z_{j}$ defined above. It is clear that $\mathfrak{X}_{0}^{+}=(0)$ and $\mathfrak{X}_{m}^{-}=\mathfrak{X}$, i.e. $F$ is a finite nest, and $F \subset \Gamma$. Note that if $Z_{j} \in F$ then $\mathfrak{X}_{j}^{+}=\mathfrak{X}_{j} \subset Z_{j} \subset \mathfrak{X}_{j+1}^{-}$. We are to prove that $\left\|T \mid Z^{\sim}\right\| \leq 4 \alpha$ for all $Z^{\sim} \in \operatorname{gap}(F)$.

The previous considerations and (3.12) show that it only remains to consider the possible gap-quotients $Z^{\sim}=\mathfrak{X}_{j}^{-} / Z_{j-1}$ for $1 \leq j \leq m$. Note that $Z_{j-1} \in \Gamma_{j}$. Then as we just have showed, the inequality

$$
\left\|T \mid\left(\mathfrak{X}_{j}^{-} / Z_{j-1}\right)\right\| \leq 2 \alpha
$$

holds in any case, i.e., if $\mathfrak{X}_{j}^{-} \in \Gamma_{j}$ or $\mathfrak{X}_{j}^{-} \in \Gamma_{j+1}$ (see (3.9) and (3.10)).

Lemma 3.4. Let $M$ be a precompact set and $\Gamma \subset$ lat $M$ be a nest. Then

$$
\varrho(M)^{2} \leq 4\|M\| \max \left\{\|M\|_{w, \Gamma},\|M\|_{\chi},\|M \mid \Gamma\|\right\} .
$$

Proof. Let $\alpha$ be the right hand side of (3.13). By Lemma 3.3, for $\varepsilon>0$ there exists a finite nest $F \subset \Gamma$ satisfying (3.7). Since

$$
\left\|M^{2}|\Gamma\|\leq\| M| \Gamma\right\|^{2} \leq\|M|\Gamma\|\cdot\| M\|\leq 4\| M\|\cdot\| M| \Gamma\|
$$

we obtain $\left\|M^{2} \mid F\right\| \leq \alpha+\varepsilon$. By Lemma 1.1, $\varrho\left(M^{2}\right)=\varrho\left(M^{2} \mid F\right)$. Hence

$$
\varrho(M)^{2}=\varrho\left(M^{2}\right)=\varrho\left(M^{2} \mid F\right) \leq\left\|M^{2} \mid F\right\| \leq \alpha+\varepsilon,
$$

and therefore $\varrho(M)^{2} \leq \alpha$.

4. Main results. Given a chain $\Gamma$ in $\mathfrak{X}$ and a subset $N \subset \operatorname{alg} \Gamma$, let $\varrho_{w, \Gamma}(N)$ denote $\lim \sup _{n \rightarrow \infty}\left\|N^{n}\right\|_{w, \Gamma}^{1 / n}$. Since $\|\cdot\|_{w, \Gamma}$ is a submultiplicative seminorm on $\operatorname{alg} \Gamma$, we may also write

$$
\varrho_{w, \Gamma}(N)=\lim _{n \rightarrow \infty}\left\|N^{n}\right\|_{w, \Gamma}^{1 / n}=\inf _{n}\left\|N^{n}\right\|_{w, \Gamma}^{1 / n} .
$$

ThEOREM 4.1. Let $M$ be a precompact set and $\Gamma \subset$ lat $M$ be a nest. Then

$$
\varrho(M)=\max \left\{\varrho_{w, \Gamma}(M), \varrho_{\chi}(M), \widehat{\varrho}(M \mid \Gamma)\right\} .
$$

In particular, if $M$ consists of weakly compact operators then

$$
\varrho(M)=\max \left\{\varrho_{\chi}(M), \widehat{\varrho}(M \mid \Gamma)\right\} \text {. }
$$


Proof. Note that (4.1) is obvious if $\varrho(M)=0$. So we assume that $\varrho(M)$ $>0$. Since $\Gamma \subset$ lat $M \subset$ lat $M^{n}$ for every integer $n>0$, we may apply Lemma 3.4 to $M^{n}$. Then

$$
\varrho\left(M^{n}\right)^{2} \leq 4\left\|M^{n}\right\| \max \left\{\left\|M^{n}\right\|_{w, \Gamma},\left\|M^{n}\right\|_{\chi},\left\|M^{n} \mid \Gamma\right\|\right\} .
$$

Hence

$$
\varrho(M)^{2}=\varrho\left(M^{n}\right)^{2 / n} \leq 4^{1 / n}\left\|M^{n}\right\|^{1 / n} \max \left\{\left\|M^{n}\right\|_{w, \Gamma}^{1 / n},\left\|M^{n}\right\|_{\chi}^{1 / n},\left\|M^{n} \mid \Gamma\right\|^{1 / n}\right\} .
$$

Taking limits as $n \rightarrow \infty$, we obtain

$$
\varrho(M)^{2} \leq \varrho(M) \max \left\{\varrho_{w, \Gamma}(M), \varrho_{\chi}(M), \varrho(M \mid \Gamma)\right\} .
$$

Taking into account that $\varrho(M \mid \Gamma) \leq \max \left\{\varrho_{\chi}(M), \widehat{\varrho}(M \mid \Gamma)\right\}$ by Lemma 2.4, we conclude that

$$
\varrho(M) \leq \max \left\{\varrho_{w, \Gamma}(M), \varrho_{\chi}(M), \widehat{\varrho}(M \mid \Gamma)\right\} .
$$

The opposite inequality is evident.

If $M$ consists of weakly compact operators then $\varrho_{w, \Gamma}(M)=0$ and (4.2) follows.

Given a complete chain $\Gamma \subset$ lat $M$, let $r(M \mid \Gamma)$ denote $\sup \{r(M \mid V)$ : $V \in \operatorname{gap}(\Gamma)\}$ (if $\Gamma$ is continuous, we set $r(M \mid \Gamma)=0$ ).

THEOREM 4.2. Let $M$ be precompact, and let $\Gamma$ be a relatively maximal nest in lat $M$. Then

$$
\begin{aligned}
\varrho(M) & =\max \left\{\varrho_{w, \Gamma}(M), \varrho_{\chi}(M), r(M \mid \Gamma)\right\} \\
& =\max \left\{\varrho_{w, \Gamma}(M), \varrho_{\chi}(M), r(M)\right\} .
\end{aligned}
$$

In particular, if $M$ consists of weakly compact operators then

$$
\varrho(M)=\max \left\{\varrho_{\chi}(M), r(M)\right\} .
$$

Proof. If $\Gamma$ is a relatively maximal nest in lat $M$ then $M \mid V$ is irreducible for every $V \in \operatorname{gap}(\Gamma)$. Since $M \mid V$ is precompact,

$$
\varrho(M \mid V)=\max \left\{\varrho_{\chi}(M \mid V), r(M \mid V)\right\}
$$

by Lemma 1.3 . Since $\varrho_{\chi}(M \mid V) \leq \varrho_{\chi}(M)$ by Lemma 2.5 and $r(M \mid V) \leq$ $r(M \mid \Gamma)$, we obtain

$$
\varrho(M \mid V) \leq \max \left\{\varrho_{\chi}(M), r(M \mid \Gamma)\right\}
$$

and therefore

$$
\widehat{\varrho}(M \mid \Gamma) \leq \max \left\{\varrho_{\chi}(M), r(M \mid \Gamma)\right\} .
$$

Since $r(M \mid \Gamma) \leq r(M)$, it follows from (4.1) that

$$
\varrho(M) \leq \max \left\{\varrho_{w, \Gamma}(M), \varrho_{\chi}(M), r(M \mid \Gamma)\right\} \leq \max \left\{\varrho_{w, \Gamma}(M), \varrho_{\chi}(M), r(M)\right\} .
$$

The opposite inequalities are evident. Now (4.4) clearly holds if $M$ consists of weakly compact operators. 
THEOREM 4.3. Let $M$ be precompact, let $Q$ be a precompact set of weakly compact operators, and let $N$ be a precompact set of compact operators (all sets are subsets of $B(\mathfrak{X}))$. Then

$$
\varrho(M \cup Q \cup N)=\max \left\{\varrho(M), \varrho_{\chi}(M \cup Q), r(M \cup Q \cup N)\right\},
$$

and in particular,

$$
\varrho(M \cup N)=\max \{\varrho(M), r(M \cup N)\} .
$$

Proof. Let $\Gamma$ be a relatively maximal nest in lat $M \cup N$. It is clear that

$$
\varrho_{w, \Gamma}(M \cup Q \cup N)=\varrho_{w, \Gamma}(M) \leq \varrho(M)
$$

and

$$
\varrho_{\chi}(M \cup Q \cup N)=\varrho_{\chi}(M \cup Q) .
$$

It follows from (4.3) that

$$
\varrho(M \cup Q \cup N) \leq \max \left\{\varrho(M), \varrho_{\chi}(M \cup Q), r(M \cup Q \cup N)\right\} .
$$

The opposite inequality is evident.

TheOREM 4.4. Let $M$ be precompact.

(i) If $M$ is commutative modulo $W(\mathfrak{X})$ then

$$
\varrho(M)=\max \left\{\varrho_{\chi}(M), r(M)\right\} .
$$

(ii) If $M$ is commutative modulo $K(\mathfrak{X})$ then

$$
\varrho(M)=r(M) \text {. }
$$

Proof. We first prove (i). Let $\Gamma$ be a relatively maximal nest in lat $M$. Since $[M, M] \subset W(\mathfrak{X})$, we have $[M, M] \subset W(\Gamma)$. Since the image of $M$ in $\operatorname{alg} \Gamma / W(\Gamma)$, say $N$, is a commutative precompact subset, we obtain $\varrho_{w, \Gamma}(M)=\varrho(N)=r(N)$ by Lemma 2.8. Since the canonical map alg $\Gamma \rightarrow$ $\operatorname{alg} \Gamma / W(\Gamma)$ is a homomorphism of Banach algebras, $r(N) \leq r(M)$. It follows from (4.3) that $\varrho(M) \leq \max \left\{\varrho_{\chi}(M), r(M)\right\}$. The opposite inequality is evident.

If $M$ is commutative modulo $K(\mathfrak{X})$, then we already have (4.7) and a similar argument shows that $\varrho_{\chi}(M)=r(M / K(\mathfrak{X})) \leq r(M)$. Therefore $\varrho(M) \leq r(M)$, and the opposite inequality is evident.

COROLlary 4.5. If $M$ is a precompact set of operators which is commutative modulo $W(\mathfrak{X})$ then, for any sequence $M_{n}$ of bounded sets of operators, tending to $M$ with respect to the Hausdorff distance,

$$
\begin{aligned}
\varrho(M) & =\max \left\{\varrho_{\chi}(M), \lim \sup \varrho\left(M_{n}\right)\right\} \\
& =\max \left\{\varrho_{\chi}(M), \liminf \varrho\left(M_{n}\right)\right\} .
\end{aligned}
$$


Proof. We should prove that

$$
\begin{aligned}
\max \left\{\varrho_{\chi}(M), \lim \sup \varrho\left(M_{n}\right)\right\} & \leq \varrho(M) \\
& \leq \max \left\{\varrho_{\chi}(M), \liminf \varrho\left(M_{n}\right)\right\} .
\end{aligned}
$$

It is not difficult to check that $\lim \sup \varrho\left(M_{n}\right) \leq \varrho(M)$. Indeed, $M_{n}^{m} \rightarrow M^{m}$ for any $m$, whence $\left\|M_{n}^{m}\right\|^{1 / m} \rightarrow\left\|M^{m}\right\|^{1 / m}$. Since $\left\|M_{n}^{m}\right\|^{1 / m} \geq \varrho\left(M_{n}\right)$, we see that $\lim \sup \varrho\left(M_{n}\right) \leq\left\|M^{m}\right\|^{1 / m}$ and it remains to take the limit as $m \rightarrow \infty$. Since $\varrho_{\chi}(M) \leq \varrho(M)$, the first inequality in (4.10) is proved.

Suppose that $\liminf \varrho\left(M_{n}\right)<\varrho(M)$ and $\varrho_{\chi}(M)<\varrho(M)$. Passing to a subsequence, and multiplying by a scalar, one may assume that $\varrho\left(M_{n}\right) \rightarrow$ $\alpha<1<\varrho(M)$ and $\varrho_{\chi}(M)<1$. It follows from Theorem 4.4(i) that $\varrho(T)>1$ for some $T \in \mathrm{SG}(M)$, say $T \in M^{k}$. Let $T_{n} \in M_{n}^{k}, T_{n} \rightarrow T$. Since $\varrho(T)>\varrho_{\chi}(T)=\varrho_{\mathrm{e}}(T), T$ has an isolated eigenvalue $\lambda$ with $|\lambda|=\varrho(T)$. By Newburgh's theorem (see Theorem 1.1.4 of [1]), $T$ is a point of continuity of the usual spectral radius, $\varrho\left(T_{n}\right) \rightarrow \varrho(T)$, whence $\varrho\left(T_{n}\right)>1$ for sufficiently large $n$. On the other hand, $\varrho\left(T_{n}\right) \leq \varrho\left(M_{n}\right)^{k} \rightarrow \alpha^{k}<1$, a contradiction.

Corollary 4.6. A precompact set $M$ of operators which is commutative modulo $W(\mathfrak{X})$ and satisfies $\varrho_{\chi}(M)<\varrho(M)$ or $\varrho(M)=0$ is a point of continuity of the joint spectral radius $\varrho$.

A simplest example of a set satisfying the hypotheses of Corollary 4.6 is a precompact set of compact operators. We recall that an operator $T \in B(\mathfrak{X})$ is a Riesz operator if $\varrho_{\mathrm{e}}(T)=0$. If $M \subset B(\mathfrak{X})$ is commutative modulo $K(\mathfrak{X})$, one also says that $M$ is an essentially commutative set of operators.

COROLlary 4.7. Any essentially commutative precompact set of Riesz operators is a point of continuity of $\varrho$.

Proof. Let $M$ be an essentially commutative precompact subset of Riesz operators. Since $M / K(\mathfrak{X})$ is a commutative precompact subset of the Calkin algebra,

$$
\varrho_{\chi}(M) \leq \varrho_{\mathrm{e}}(M)=\varrho(M / K(\mathfrak{X}))=r_{\text {sup }}(M / K(\mathfrak{X}))
$$

by Lemma 2.8. As $M / K(\mathfrak{X})$ consists of quasinilpotents, $r_{\sup }(M / K(\mathfrak{X}))=0$. Hence $\varrho_{\chi}(M)=0$ and, by Corollary $4.6, M$ is a point of continuity of $\varrho$.

Let $A(M)$ denote the closed subalgebra generated by $M \subset B(\mathfrak{X})$.

Corollary 4.8. Let $G$ be an essentially commutative semigroup of quasinilpotent operators. Then $\varrho(M)=0$ for every precompact subset $M \subset$ $A(G)$.

Proof. Note that $\varrho(N)=r(N)$ for every precompact subset $N \subset G$ by Theorem 4.4(ii). Since the semigroup generated by $N$ consists of quasinilpotents, $r(N)=0$ and therefore $\varrho(N)=0$. Let $B$ be the subalgebra generated by $G$. If $Q$ is a finite subset of $B$, then $\varrho(Q)=0$ because $Q$ is the set of 
polynomials in elements of some finite subset $N \subset G$ (see [11], Corollary $2.10)$.

Let $M$ be a precompact subset of $A(G)$. Since $A(G)$ is an essentially commutative algebra of Riesz operators, $M$ is a point of continuity of $\varrho$ by Corollary 4.7. There exists a sequence $\left(Q_{n}\right)$ of finite subsets of $B$ which tends to $M$ with respect to the Hausdorff distance. Since $\varrho\left(Q_{n}\right)=0$, we obtain $\varrho(M)=0$.

Here we list some extensions of our results; the proofs need some auxiliary technique and will be published elsewhere.

(1) The Berger-Wang formula, $\varrho(M)=r(M)$, is valid for precompact subsets of a Banach algebra if $M$ consists of compact elements. Recall that an element $a$ of a Banach algebra $A$ is called a compact element of $A$ if the map $x \mapsto a x a, x \in A$, is compact.

(2) The Berger-Wang formula is valid for finite subsets of a postliminal $C^{*}$-algebra.

(3) The commutativity conditions modulo $W(\mathfrak{X})$ (or $K(\mathfrak{X})$ ) in Theorem 4.4 can be considerably weakened: one may suppose only that $M / W(\mathfrak{X})$ (or $M / K(\mathfrak{X})$ ) belongs to the closed associative subalgebra generated by a nilpotent Lie subalgebra.

Other applications to Banach algebras will also be published separately.

\section{References}

[1] B. Aupetit, Propriétés spectrales des algèbres de Banach, Lecture Notes in Math. 735, Springer, Berlin, 1979.

[2] M. A. Berger and Y. Wang, Bounded semigroups of matrices, Linear Algebra Appl. 166 (1992), 21-27.

[3] D. Colella and C. Heil, The characterization of continuous, four-coefficient scaling functions and wavelets, IEEE Trans. Inform. Theory, Special Issue on Wavelet Transforms and Multiresolution Signal Analysis, 38 (1992), 876-881.

[4] I. Daubechies and J. Lagarias, Two-scale difference equations: I. Existence and global regularity of solutions, SIAM J. Math. Anal. 22 (1991), 1388-1410.

[5] M. M. Day, Normed Linear Spaces, Springer, Berlin, 1958.

[6] P. S. Guinand, On quasinilpotent semigroups of operators, Proc. Amer. Math. Soc. 86 (1982), 485-486.

[7] V. Lomonosov, Invariant subspaces for operators commuting with compact operators, Functional Anal. Appl. 7 (1973), 213-214.

[8] V. Yu. Protasov, The joint spectral radius and invariant sets of linear operators, Fundament. Prikl. Mat. 2 (1996), 205-231 (in Russian).

[9] —, The generalized joint spectral radius. Geometric approach, Izv. Ross. Akad. Nauk Ser. Mat. 61 (1997), no. 5, 99-136 (in Russian).

[10] G.-C. Rota and W. G. Strang, A note on the joint spectral radius, Indag. Math. 22 (1960), 379-381. 
[11] V. S. Shulman and Yu. V. Turovskiı̌, Joint spectral radius, operator semigroups and a problem of W. Wojtyński, J. Funct. Anal. 177 (2000), 383-441.

[12] Yu. V. Turovskiı̌, Volterra semigroups have invariant subspaces, ibid. 162 (1999), 313-323.

Department of Mathematics

Institute of Mathematics and Mechanics

Vologda State Technical University

9 F. Agaev St.

15 Lenin St., Vologda 160000, Russia

Baku 370141, Azerbaijan

E-mail: shulman_v@yahoo.com

E-mail: info@sinam.net

and

School of Mathematical Sciences

University of North London

166-220 Holloway Rd.

London N7 8DB, UK

Received August 28, 2000 